\title{
Evidence of underdiagnosis of myasthenia gravis in older people
}

\author{
A Vincent, L Clover, C Buckley, J Grimley Evans, P M Rothwell, and the UK Myasthenia \\ Gravis Survey
}

J Neurol Neurosurg Psychiatry 2003;74:1 105-1 108

See end of article for authors' affiliations

Correspondence to Professor Angela Vincent, Weatherall Institute of Molecular Medicine, John Radcliffe Hospital, Oxford OX3 9DS, UK; angela.vincent@ imm.ox.ac.uk

Received 1 August 2002 Accepted

22 October 2002

\begin{abstract}
Background: Myasthenia gravis is a potentially serious but treatable muscle disease caused by autoantibodies directed at the acetylcholine receptor (AChR) on the postsynaptic membrane of the neuromuscular junction. There is anecdotal evidence that the diagnosis is sometimes missed in older patients.

Objective: To examine the incidence and age distribution of positive AChR antibodies in samples referred to diagnostic laboratories in the UK, and the prevalence of positive AChR antibodies in samples from a cohort of older individuals.

Methods: Positive AChR antibody tests were identified from all UK centres registered for the assay with the European quality assurance scheme (EQAS) during 1997-99, and the age and sex specific incidence was calculated, based on the UK population. The prevalence of AChR antibodies in sera from a sample of 2000 individuals aged $\geqslant 60$ years was determined.

Results: 3183 individuals had positive AChR antibody tests on routine screening during the years 1997 to 1999 in the UK, giving an annual incidence of 1.8/100 000. In both sexes, the age specific incidence rose steeply between the ages of 45 and 74, reaching 9.9/100 000 in men, and then fell, with a sharp decline above the age of 80 . In the prevalence study, whereas only one serum from individuals aged $60-74$ years was positive for AChR antibodies $(0.12 \%)$, sera from eight individuals aged $\geqslant 75$ years were positive $(0.7 \%)$. Only one had a previous clinical diagnosis of myasthenia gravis but four others had histories of stroke or transient ischaemic attacks.

Conclusions: The sharp fall in the incidence of clinically recognised myasthenia gravis in people over 80 years of age in our national AChR antibody incidence study, and the high prevalence of previously unrecognised positive AChR antibodies in those $\geqslant 75$ years old, suggest that myasthenia gravis may be substantially underdiagnosed in older people.
\end{abstract}

M yasthenia gravis is a potentially serious but treatable muscle disease caused by autoantibodies directed at the acetylcholine receptor (AChR) on the postsynaptic membrane of the neuromuscular junction. ${ }^{12}$ It is generally thought to affect around 10-15 per 100000 of the population and to be most common in young women..$^{1-3}$ Such patients present during early adult life, often have enlarged thymuses with numerous germinal centres, tend to improve after thymectomy, and are likely to have the HLA B8, DR3 antigen. ${ }^{3}$ Less commonly $(<10 \%$ of the total), the disease occurs in patients with thymic tumours.

Myasthenia gravis can also present in middle age or later. In this situation, the thymus is not usually abnormal, there is only a weak association with HLA B7 DR2 antigen, and the sex ratio is nearer unity. ${ }^{4}$ It is now recognised that the incidence and prevalence of myasthenia gravis in middle aged and older patients are greater than was previously thought. ${ }^{5-12}$ However, the clinical features tend to be different from those in younger cases, with more bulbar involvement in older people, and the differential diagnosis is wider. Consequently, myasthenia gravis in later life is sometimes mistaken for other conditions, such as stroke, ${ }^{1314}$ motor neurone disease, or parkinsonism, and may be underdiagnosed.

In two previous studies, an apparent fall in the incidence of AChR antibodies in elderly people has been shown, ${ }^{5}$ but neither study discussed the possibility of underdiagnosis in later age groups. To determine whether the disease is underdiagnosed in older people we undertook two studies. First, to detect any fall off in diagnosis at later ages, we calculated the age and sex specific incidence of positive AChR antibody tests in the UK by collaboration with all UK centres that are regis- tered with EQAS (the European quality assurance scheme) for AChR antibody assays. A national multicentre study was required in order to have sufficient statistical power to determine the age and sex specific incidence in later life with narrow confidence intervals. Second, we tested sera from a community based sample of 2000 individuals aged 60 years and over, from the Oxford region, for AChR antibodies.

\section{METHODS}

\section{Assay data}

We obtained data on 3836 individuals who tested positive for AChR antibody at the nine British centres that are registered with EQAS during three consecutive years (1997 to 1999). All use a standard immunoprecipitation test with ${ }^{125} \mathrm{I}-\alpha-$ bungarotoxin labelled human AChR. ${ }^{15}{ }^{16}$ These laboratories perform the vast majority of tests in the UK and Northern Ireland, and we are not aware of any tests that are routinely sent to laboratories outside the UK. We excluded all results from individuals with the same name and date of birth, referrals from foreign laboratories, and tertiary referrals of previously diagnosed cases to Professor John Newsom-Davis in Oxford. In the 3183 remaining cases (Birmingham 1276, Oxford 989, Sheffield 302, Liverpool 164, St George's London 112, Glasgow 138, Bristol 100, Cardiff 53, Nottingham 49), we studied age and sex at the time of sampling. Twelve were from babies with neonatal myasthenia gravis and were excluded from further analysis. In 2795 cases (87.8\%) an AChR antibody titre was also available. 

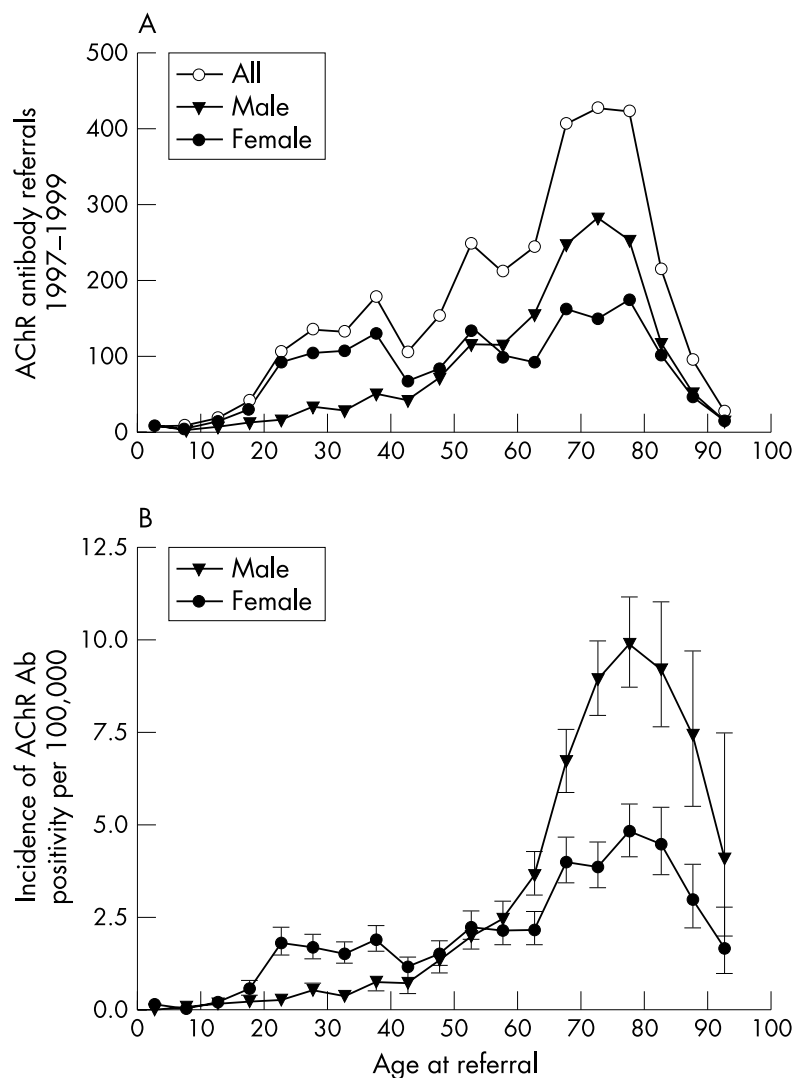

Figure 1 Total numbers of positive acetylcholine receptor (AChR) antibody titres in the UK from 1997 to 1999 inclusive, with data for men and women also shown separately $(A) ; 12$ positive results from babies with probable neonatal myasthenia have been omitted. (B) Age and sex specific incidence of positive AChR antibody in the UK from 1997-99 inclusive.

\section{Estimation of age and sex specific incidence}

Given the high specificity of the AChR antibody assay, ${ }^{15}{ }^{16}$ the fact that the test is rarely requested without clinical suspicion of myasthenia gravis, and that it is routine practice to confirm clinical diagnoses by antibody testing, we considered it reasonable to use the national AChR antibody data to estimate the likely incidence of clinically diagnosed cases of myasthenia gravis. We assumed that repeat testing following initial diagnosis was rare, and that the vast majority of positive titres reported during our three year study period represented incident cases. This assumption was supported by analysis of all tests submitted to the Oxford laboratory from 1994 to 1999; less than $5 \%$ of the positive results identified during 1997 to 1999 were from patients diagnosed during 1994 to 1996. Assuming, therefore, that we had an accurate assessment of all positive AChR antibody results in Britain from 1997 to 1999, and that these were incident cases, we used the age and sex stratified mid-year population estimates for England, Wales, Northern Ireland, and Scotland for 1998 to calculate the age and sex specific incidence of AChR antibody positivity in Britain for the period 1997 to 1999. Calculation of 95\% confidence intervals (CI) for the incidence rates assumed that the number of cases in each age group followed a Poisson distribution.

\section{Cohort of older people}

Blood samples were obtained from the Oxford healthy ageing project, a module of the Medical Research Council cognitive function and aging study (CFAS).${ }^{17}$ Potential participants in the Oxford healthy aging project were nominated as a systematic sample from the primary care registers of all people aged
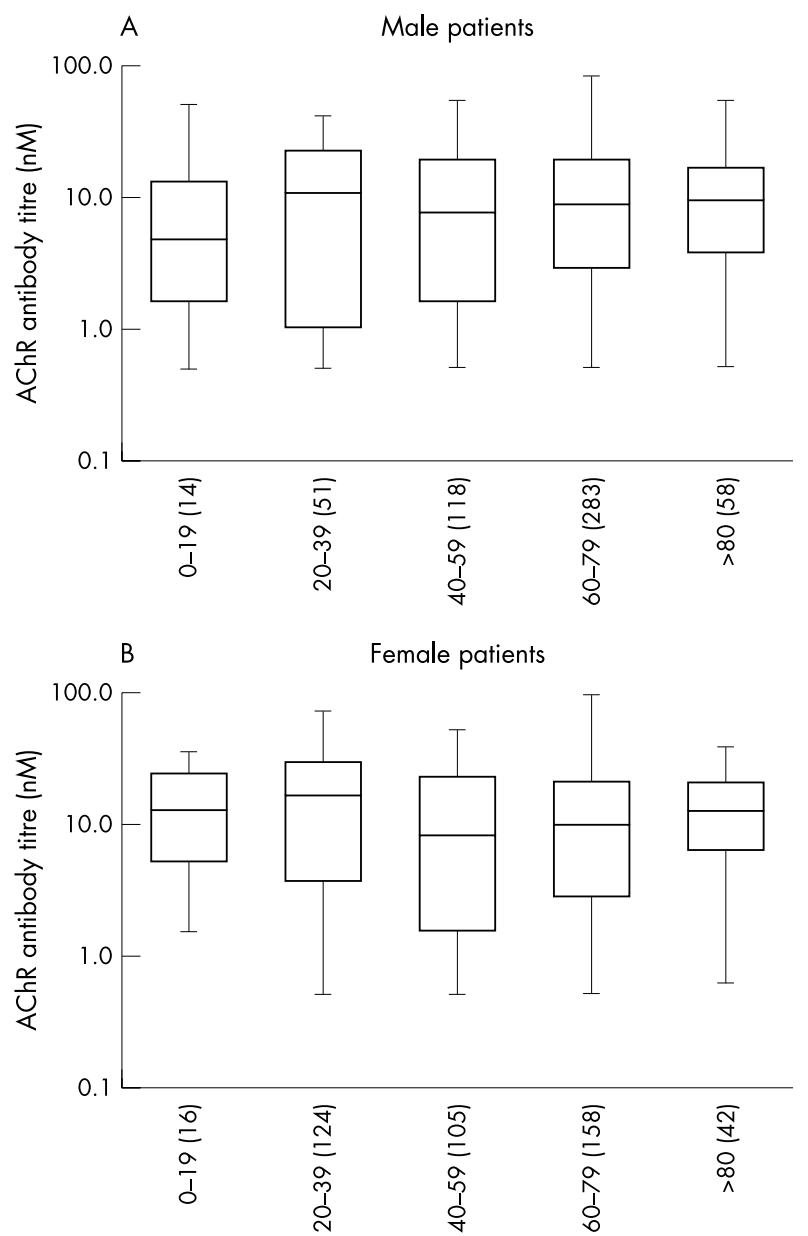

Age range (No of samples)

Figure 2 Acetylcholine receptor (AChR) antibody values measured in Oxford for men (A) and women (B) show that similar high values are found at all ages and in both sexes.

65 and over, resident in the four central postal districts of Oxford. From a sampling frame of 3555 individuals, 2740 potential participants agreed to be interviewed. The 1649 blood samples were from those who consented to the initial research interview, which included clinical data, and who, two years later, consented to donate a blood sample. The specimens were obtained and stored at $-80^{\circ} \mathrm{C}$. We also analysed sera from a further 351 patients aged 60 and over attending outpatient clinics with non-neurological complaints at the John Radcliffe Hospital between October 1999 and January 2000. The sera were screened by the immunoprecipitation assay, as used in routine diagnostic testing, and all positive sera were retested to confirm AChR antibody specificity. The Oxford healthy aging project and the associated blood sampling and anonymised analysis were approved by the Central Oxford research ethics committee.

\section{RESULTS}

\section{AChR antibodies in referred samples}

The age at referral of the 3171 positive AChR antibody results from the UK is shown in fig lA. The age and sex distribution was consistent across the centres (Birmingam $v$ Oxford $v$ others), and for each of the three years of study (data not shown). The overall incidence was 1.8 cases/100 000 per year. There was the expected high female to male ratio in younger patients, presenting mainly between 15 and 40 years of age, and an increasing number of both men and women presenting 
Table 1 Demographic characteristics of 1147 individuals aged $\geqslant 75$ years

\begin{tabular}{|c|c|c|c|c|c|c|}
\hline & \multicolumn{6}{|c|}{ Age (years) } \\
\hline & 75 to 79 & 80 to 84 & 85 to 89 & 90 to 94 & $>94$ & Total \\
\hline Men & 189 & 156 & 89 & 20 & 5 & 459 \\
\hline Women & 238 & 248 & 147 & 43 & 12 & 688 \\
\hline Total & 427 & 404 & 236 & 63 & 17 & 1147 \\
\hline $\begin{array}{l}\text { Positive AChR } \\
\text { antibodies }\end{array}$ & $3 F$ & $2 F$ & $2 F$ & & $1 M$ & 8 \\
\hline
\end{tabular}

Values are numbers of individuals.

AChR, acetylcholine receptor; $F$, female; $M$, male.

\begin{tabular}{lll} 
Table 2 & Acetylcholine receptor (AChR) antibody positive individuals from among \\
1147 subjects aged $\geqslant 75$ years & \\
\hline Age, sex & AChR antibody (nM) & Reported clinical conditions \\
\hline $84, \mathrm{~F}$ & 0.9 & TIA, stroke \\
$88, \mathrm{~F}$ & 0.9 & TIA, angina \\
$82, \mathrm{~F}$ & 1.0 & Nil \\
$76, \mathrm{~F}$ & 1.9 & Eczema \\
$75, \mathrm{~F}$ & 3.1 & Myasthenia gravis, hypertension, psoriasis \\
$78, \mathrm{~F}$ & 3.2 & Gallstones, hiatus hernia \\
$88, \mathrm{~F}$ & 3.9 & Stroke, falls \\
$95, \mathrm{M}$ & 13.5 & TIA, LVF, anaemia \\
\hline
\end{tabular}

$F$, female; LVF, left ventricular failure; $M$, male; TIA, transient ischaemic attacks.

over 45 years of age, the number of cases peaking between 70 and 80 years and then falling abruptly after age 80 (fig 1A).

In order to calculate the age specific incidence of AChR antibody positivity, the incident data need to be adjusted for the total number of individuals in each age group. In both men and women, the age specific annual incidence (fig 1B) rose sharply between the ages 45 and 74, reaching 4.8 (95\% CI, 4.1 to 5.5$)$ per 100000 in women and 9.9 (95\% CI, 8.7 to 11.2 ) per 100000 in men at age 75-79 years. However, in both sexes, this trend reversed above age 75-79, and the age specific incidence rates subsequently fell sharply, dropping to 1.6 (95\% CI, 0.9 to 2.7) per 100000 in women and 4.0 (95\% CI, 1.9 to 7.4 ) per 100000 in men at age 90-94 years (fig 1B).

It was possible that the positive AChR antibody values in older individuals were of less clinical significance than those in younger patients. Figure 2 shows the mean AChR antibody values plotted for different ages at referral for men and women from the Oxford laboratory. Results from other centres were similar (data not shown). Although, as in all studies of AChR antibodies, the range of values is very wide, there was no trend towards lower values with increasing age.

\section{AChR antibodies in cohort of older people}

Sera from nine of the 2000 individuals aged 60 years and over were positive for AChR antibodies. However, because the results in fig 1 suggested that there might be underreferral of samples after age 75 , we separated the samples into those from individuals aged $60-74$ and those aged $\geqslant 75$ years. Table 1 shows the age and sex distribution of the 1147 individuals aged $\geqslant 75$ years. Whereas only one of the 853 individuals aged 60-74 years $(0.12 \%)$ was positive for AChR antibodies, eight of those aged $\geqslant 75$ years were positive $(0.71 \%$ ) (7/980 in the community based cohort and 1/167 from the outpatient cohort). All but one of the values were at the lower end of the range, but all were compatible with a clinical diagnosis of myasthenia gravis. The AChR antibody values, and clinical data available from the patient records, are summarised in table 2. One individual had had a clinical diagnosis of myasthenia gravis, but no diagnosis of myasthenia gravis had been made in the other seven people with positive AChR antibodies. Notably, however, four of them had a history of stroke or transient ischaemic attacks.

\section{DISCUSSION}

This national study is the largest ever of the incidence of AChR antibodies. The results confirm previous studies showing a high incidence of diagnosed myasthenia gravis in older people..$^{5-12}$ In addition, the large size of our study allowed us to determine the age and sex specific incidence of the disease with narrow confidence intervals in the very old. Although the incidence increased steeply in both sexes between the ages of 45 and 79 years, achieving 9.9 per 100000 in men, it then fell sharply at later ages. This suggests either that myasthenia gravis has a temporary peak in incidence in the older age groups, which then falls off, or that the diagnosis is being missed in many older individuals, particularly in those over 80 years of age. To test our conclusion that there are significant numbers of elderly people with undiagnosed positive AChR antibodies, we studied 2000 individuals aged 60 and over. We found positive AChR antibodies in eight of the 1147 individuals who were $\geqslant 75$ years (prevalence of one in 141 ), compared with only one in the 853 individuals between 60 and 74 years. Thus the results of both our national incidence study and our community based prevalence study suggest that underdiagnosis of myasthenia gravis may be common in the elderly, and highlight the need for a high index of suspicion in older patients for this treatable disease.

The most striking observation was that the incidence peaked in 75-79 year old men and women and then fell off in people over 80 years old, suggesting underdiagnosis in the very elderly. We could have overestimated the incidence of positive AChR antibody tests in the UK because we could not be certain that all of the tests that we studied were incident cases. However, we took pains to avoid this (Methods), and as we were mainly interested in the age specific pattern rather than the absolute incidence, any overestimation is likely to have applied fairly equally at all ages, and would not explain the steep fall in later life. It could be that we are seeing a "cohort" effect, whereby people born at a certain time, in the early to mid 1920s, had a particular environmental exposure that increased their risk of developing myasthenia gravis. Although a very interesting possibility, the rise and fall in incidence is so steep that it would have required a very short period of exposure, a short period of susceptibility to the exposure (for example, in utero or at the age of 0 to 5 years), 
and a very long and relatively fixed incubation period (for example, $70 \pm 5$ years). This seems unlikely given that studies from other countries like Greece ${ }^{11}$ have seen a similar peak. Nevertheless, another analysis in, say, 10 years time could be undertaken to address this possibility. Alternatively, the apparent fall off in incidence in the very elderly might reflect competing risks if those who are at risk of developing myasthenia gravis are also at a higher risk than the general population of developing some other, fatal disease. However, for the same reasons given above, the incidence of this disease would have to be extremely strongly associated with the risk of developing myasthenia gravis, and would have to be confined mainly to people in their 80 s and 90 s.

The overall incidence rate of $1.8 / 100000$ that we found is higher than the value of $0.74 / 100000$ in the recent Greek study, ${ }^{11}$ which also identified incident cases by a positive AChR antibody test. They found a point prevalence of 17.5/100 000 in individuals aged $\geqslant 70$ years, which is threefold greater than the prevalence of about 5/100 000 in individuals aged $\geqslant 80$ years in a recent UK survey. ${ }^{9}$ We have not attempted to calculate the likely prevalence in elderly individuals based on our incidence figures, but one would predict a value higher than either of these studies.

The frequency of positive AChR antibodies in our prevalence study was very surprising. One possible concern is that the high prevalence in individuals aged $>74$ years was part of a non-specific increase in autoantibodies with age, and was not of clinical significance. However, this is not supported by the fact that these sera were negative for all other autoantibodies that we examined in parallel (voltage gated calcium and potassium channels, glutamic acid decarboxylase, $\mathrm{Hu}$ and Yo antigens) (Clover L, Vincent A, in preparation), and only one of the eight was positive for thyroid antibodies. Moreover, although false positive AChR antibody results can occur in patients on penicillamine treatment, in patients with thymic tumours, and in the monozygotic twin siblings of patients with myasthenia gravis, the assay is otherwise highly specific $(>99.9 \%$ ) and is thought to be diagnostic of the disease. ${ }^{15}$ Further studies are now required to confirm these striking findings in a prospective study, and to investigate their clinical relevance.

Underdiagnosis of myasthenia gravis could be explained at least in part by the fact that many of the presenting symptoms (fatigue, muscle weakness, slurred speech, and difficulty in swallowing) have a broad differential diagnosis in older people. For example, myasthenia gravis is sometimes misdiagnosed as stroke ${ }^{13}{ }^{14}$ or motor neurone disease (Vincent A, unpublished observations). It is noteworthy that four of the cases in our elderly cohort who had positive AChR antibodies and had not been given a diagnosis of myasthenia gravis had a diagnosis of stroke or transient ischaemic attacks (table 2). Unfortunately, under the terms of the ethics approval for the Oxford healthy aging study, we were unable to re-examine any of the AChR antibody positive individuals for overt or subclinical myasthenia gravis. Such a study needs to be done in future cohorts.

There is also evidence of possible underdiagnosis of other uncommon neurological conditions in older people. For example, misdiagnosis and underinvestigation have been suggested as the cause of the apparent sharp fall in incidence of motor neurone disease in late life. ${ }^{18} 19$ Much has been written about the importance of not discriminating against older people in relation to the provision of treatment. ${ }^{20-22}$ It is important also that the same principles are applied to referral for specialist investigation and diagnosis.

\section{ACKNOWLEDGEMENTS}

We are very grateful to Nick Willcox and John Newsom-Davis FRS for their input into the early stages of this study and their helpful comments. PMR is supported by the MRC. LC was supported by the Myasthenia Gravis Association and the National Lottery Charities Board. The Oxford Healthy Aging Project (OHAP) is a module of the MRC Cognitive Function and Aging Study (MRC-CFAS), and is supported by grants from the MRC, the Department of Health, and the Clothworkers' Foundation.

\section{Authors' affiliations}

A Vincent, L Clover, C Buckley, P M Rothwell, Department of Clinical Neurology and Weatherall Institute of Molecular Medicine, University of Oxford, Oxford, UK

J Grimley Evans, Division of Clinical Geratology, Nuffield Department of Medicine, University of Oxford, Radcliffe Infirmary, Oxford

UK Myasthenia Gravis Survey: I Hart (Liverpool), A Huisson (Nottingham), K Mees (Birmingham), J Miles (St George's, Tooting), A Milford Ward (Sheffield), N Robertson (Cardiff), W Taylor (Oxford), J Vetch (Glasgow), P Virgo (Bristol), H Willison (Ǵlasgow)

Competing interests: none declared

\section{REFERENCES}

1 Vincent A, Palace J, Hilton-Jones D. Myasthenia gravis. Lancet 2001;357:2122-8.

2 Oosterhuis HJGH. Clinical aspects and epidemiology. In: Myasthenia gravis. Groningen: Neurological Press, 1997:17-48.

3 Compston DAS, Vincent A, Newsom-Davis J, et al. Clinical, pathological, HLA antigen and immunological evidence for disease heterogeneity in myasthenia gravis. Brain 1980;103:579-601.

4 Aarli JA. Late-onset myasthenia gravis: a changing scene. Arch Neurol 1999;56:25-7.

5 Somnier F. Myasthenia gravis. Danish Med Bull 1996:43:1-10.

6 Sanders DB, Andrews PI, Howard JF, et al. Seronegative myasthenia gravis. Neurology 1997;48(suppl 5):S40-5.

7 Slesak G, Melms A, Gerneth F, et al. Late-onset myasthenia gravis. Follow-up of 113 patients diagnosed after age 60. Ann NY Acad Sci 1998:841:777-80.

8 Schon $F$, Drayson M, Thompson RA. Myasthenia gravis and elderly people. Age Ageing 1996:26:56-8.

9 Robertson NP, Deans J, Compston DAS. Myasthenia gravis: a population based epidemiological study in Cambridgeshire, England. J Neurol Neurosurg Psychiatry 1998;65:492-6.

10 Morino AG, Gragnani F, Fiorelli M. Myasthenia in the elderly: a hospital based study. Acta Neurol Scand 1996;93:260-2.

11 Poulas K, Tzibri E, Kokla A, et al. Epidemiology of seropositive myasthenia gravis in Greece. J Neurol Neurosurg Psychiatry 2001:71:352-6.

12 MacDonald BK, Cockerell OC, Sander JW, et al. The incidence and lifetime prevalence of neurological disorders in a prospective community-based study in the UK. Brain 2000;1 23:685-76.

13 Kleiner-Fisman G, Kott HS. Myasthenia gravis mimicking stroke in elderly patients. Mayo Clin Proc 1998;73:1077-8.

14 Kluin KJ, Bromberg MB, Feldman EL, et al. Dysphagia in elderly men with myasthenia gravis. J Neurol Sci 1996;138:49-52.

15 Vincent A, Newsom-Davis J. Acetylcholine receptor antibody as a diagnostic test for myasthenia gravis: results in 153 validated cases and 2967 diagnostic assays. J Neurol Neurosurg Psychiatry 1985;48: 1246-52

16 Lindstrom JM, Seybold ME, Lennon VA, et al. Antibody to acetylcholine receptor in myasthenia gravis. Prevalence, clinical correlates and diagnostic value. Neurology 1976;26:1054-9.

17 MRC CFAS. Medical Research Council cognitive function and ageing study. Cognitive function and dementia in six areas of England and Wales: the distribution and prevalence of GMS organicity level in the MRC CFA Study. Psychol Med 1998;28:319-35.

18 Kahana E, Alter M, Feldman S. Amyotrophic lateral sclerosis, a population study. J Neurol 1976;212:205-13.

19 Scottish Motor Neuron Disease Research Group. The Scottish Motor Neuron Disease Register: a prospective study of adult onset motor neuron disease in Scotland. Methodology, demography and clinical features of incident cases in 1989. J Neurol Neurosurg Psychiatry 1992;55:536-41.

20 Bowling A. Ageism in cardiology. BM 1999;319:1353-5

21 Grant PT, Henry JM, McNaughton GW. The management of elderly blunt trauma victims in Scotland: evidence of ageism? Injury 2000;31:519-28.

22 Kearney N, Miller M. Elderly patients with cancer: an ethical dilemma. Crit Rev Oncol Hematol 2000;33: 149-54. 